ORIGINAL ARTICLE

\title{
Effects of six weeks of detraining on retention of functional fitness of old people after nine weeks of multicomponent training
}

\author{
N F Toraman, N Ayceman
}

Br J Sports Med 2005;39:565-568. doi: 10.1136/bjsm.2004.015586

See end of article for authors' affiliations .....................

Correspondence to: Dr Toraman, Akdeniz University School of Physical Education and Sports, Health Sciences, Antalya 07058, Turkey; ftoraman@akdeniz.edu.tr

Accepted 7 December 2004
Objectives: To examine the effects of age on functional fitness after six weeks of detraining. Methods: Elderly subjects, aged 60-86 years, completed a nine week multicomponent exercise training programme. They performed the senior fitness test every two weeks during the six week detraining period, and the responses of 12 young-old subjects ( $\mathrm{YO}$, aged 60-73 years) and nine older subjects $(\mathrm{O}$, aged 7486 years) were compared.

Results: Functional fitness improved during the exercise training period. Performances in the chair stand and six minute walk for the $O$ group had significantly declined compared with post-training values after two weeks of detraining $(p<0.01)$, whereas there were no significant changes in the YO group. Scores on the functional fitness tests declined further between two and four weeks of detraining in both of the groups $(p<0.01)$. In the YO group, there were significant losses in performance on the chair stand, chair sit and reach, and six minute walk tests, and in the $O$ group on the chair stand and up and go tests after six weeks of detraining compared with after four weeks of detraining $(p<0.01)$. The components of functional fitness most affected by detraining were lower extremity flexibility after two and four weeks of detraining, and agility/dynamic balance after six weeks of detraining.

Conclusion: Changes in lower extremity flexibility, up and go, and six minute walk performances in response to six weeks of detraining are affected by age in elderly adults.
$\mathrm{F}$ unctional fitness is defined as having the physical capacity to perform normal everyday activities safely and independently without undue fatigue and includes components such as lower and upper body muscle strength, lower and upper body flexibility, aerobic endurance, and motor agility/dynamic balance. ${ }^{1}$ The percentage decline in the functional fitness items is generally consistent with age related declines in physical performance. ${ }^{2}$

Despite studies suggesting that training helps to attenuate the effects of aging on functional fitness, ${ }^{3-5}$ it is not known for how long these beneficial effects are maintained. Detraining often occurs in previously sedentary people who participate in exercise for several weeks or months and then stop. ${ }^{6}$ Most studies of detraining in elderly people have reported only partial loss of the gains in muscular strength achieved during training. ${ }^{7-14}$ However, one study reported a return to pre-training strength after one year of detraining, ${ }^{15}$ and another showed an even greater decline to a level below pre-training values. ${ }^{16}$ In contrast, gains in cardiovascular fitness last longer in elderly people. ${ }^{7}$ Furthermore, few studies have examined the effects of detraining on functional capacity in elderly people. ${ }^{10} 121516$

Therefore the purposes of this study were to evaluate the effects of six weeks detraining on functional fitness in youngold ( $\mathrm{YO}$, aged 60-73 years) and older ( $\mathrm{O}$, aged 74-86 years) subjects, and to determine whether functional fitness responded differently to detraining in these age groups.

\section{METHODS}

\section{Participants}

Forty two elderly adults were initially recruited to take part in a randomised trial designed to investigate the effects of a nine week exercise training programme on functional fitness and body composition ${ }^{3}$ and the age responses to the multicomponent training. ${ }^{4}$ Subjects were volunteers who were older than 60 years, healthy, and without serious cardiovascular or musculoskeletal diseases, living independently in a retirement home, performing activities of daily living without mobility aids, and had a standardised mini-mental state examination score $\geqslant 20$. Twenty two subjects were assigned to the YO group (aged 60-73 years), and 20 to the O group (aged 74-86 years). The YO and $\mathrm{O}$ groups were randomly subdivided into exercisers and control subjects, who did not exercise. Twelve of the YO group participated in the exercise programme and 10 served as non-exercise control subjects; nine of the $\mathrm{O}$ group exercised and 11 were controls.

The elderly subjects provided written informed consent before the training and detraining. Of the 42 subjects in the initial phase of the study, 21 of the exercisers and four of the control subjects volunteered for the detraining study. Because the aim of this study was to investigate the effects of detraining after a training programme, only the values for the exercise group were analysed. All participants had medical clearance to participate in the testing, training, and detraining sessions. Normal activities of daily living were maintained during detraining, but none of the subjects participated in any exercise programme.

\section{Intervention}

The first phase of the study has been described in detail elsewhere. ${ }^{3}$ Briefly, participants performed aerobic, resistance, and flexibility exercise training under the direct supervision of a research assistant. The training variables in the first week's aerobic training were: intensity, 50\% of heart rate reserve; duration, 20 minutes per session; and frequency, three days a week. After the first week, the aerobic training duration was increased by five minutes and the intensity by $5 \%$ of heart rate reserve every two weeks. Dynamic resistance exercises were performed in a circuit, organised as a row of 10 resistance exercise stations. The resistance exercises included 


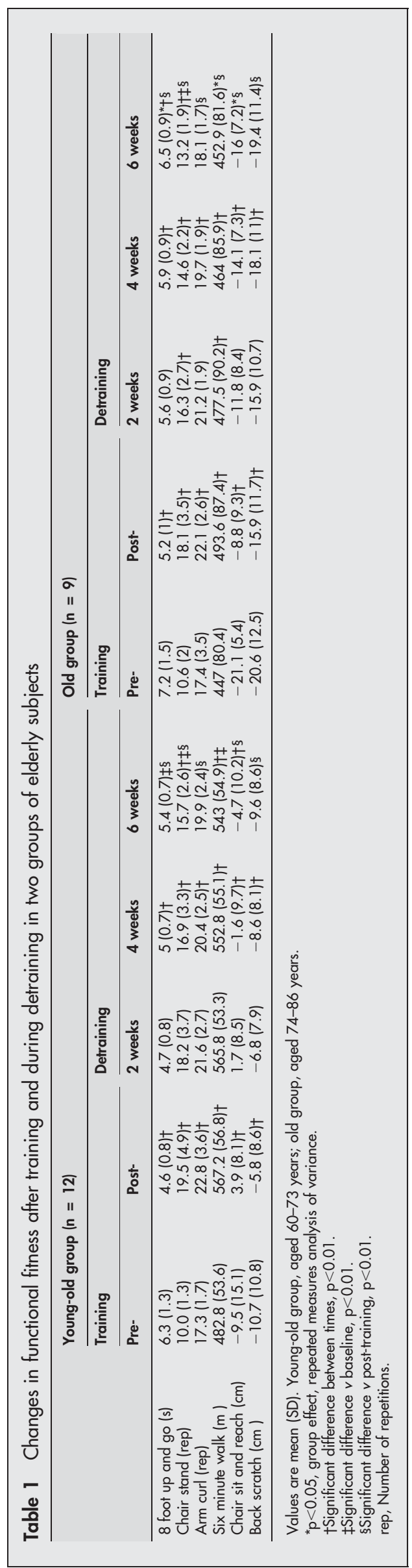

stair stepping, knee flexion, seated lower leg lift, arm raise, chair squat, biceps curl, toe raise, modified push up, abdominal crunch, and hip extension. In the first week, subjects performed a single set of eight repetitions of unloaded exercises; the number of repetitions increased to 12 in the second week, and the number of sets increased to three in the third week. Resistance training began at $50 \%$ of the predicted one repetition maximum, and the load gradually increased to $80 \%$ of the predicted one repetition maximum. When the number of repetitions was increased, the weight remained constant. The flexibility programme included static stretching of the major muscle-tendon groups. Subjects stretched in the middle of each set and after each session. There were no significant differences at baseline between the groups $(p>0.05)$. The nine week multicomponent training programme significantly increased performance on functional fitness tests in both the YO and O groups, and the rate of restoration of functional fitness was similar in the two groups. ${ }^{4}$

\section{Measurements}

Both groups were tested before training (pre-training) and after training (post-training) and every two weeks. On the test day, subjects first completed a 10 minute warm up led by an exercise instructor and then completed the senior fitness test items, ${ }^{1}$ as validated by Rikli and Jones. ${ }^{17}$

The senior fitness test consists of six assessment items. The chair stand test assesses lower body strength. Each subject completed two practice repetitions and one 30 second test trial. The score was the total number of stands executed correctly within 30 seconds. The arm curl test assesses upper body strength. Each subject completed two practice repetitions and one 30 second test trial. The score was the total number of hand weight curls through the full range of motion in 30 seconds. The chair sit and reach test assesses lower body flexibility. Each subject completed two practice trials and two test trials. The score was the best distance achieved between the extended fingers and the tip of the toe. The back scratch test assesses upper body flexibility. Each subject completed two practice trials and two test trials. The score was the best distance achieved between the extended middle fingers. The 8 foot up and go test assesses agility and dynamic balance. Each subject completed one practice trial and two test trials. The score was the shortest time to rise from a seated position, walk 8 feet, turn, and return to the seated position. The six minute walk test assesses aerobic endurance. Each subject completed one practice trial two days before the test and one test trial. The score was the total distance walked in six minutes along a $45.72 \mathrm{~m}$ rectangular course, which was marked every $4.57 \mathrm{~m}$.

\section{Statistical analysis}

All data are presented as means (SD). Data were analysed using SPSS software (version 10.0). Student's $t$ test for independent samples was used to compare baseline values between the groups. Two way, repeated measures analysis of variance was performed to determine changes over time for functional fitness tests. The level of significance was set at 0.05 .

Post hoc analyses were conducted using paired $t$ tests to examine within-group differences between baseline values and those after nine weeks of training and after every two weeks of detraining. The Bonferroni correction was applied to set the significance criterion to 0.01 - that is, 0.05 divided by 5 .

To estimate whether changes in the parameters were meaningful in a practical way, the standardised response means (mean changes divided by the SD) were measured to calculate effect size; an effect size of 0.2-0.49 was considered small, $0.5-0.79$ moderate, and 0.8 or greater, large. 
Table 2 Within-group and between-group effect sizes after two, four, and six weeks of detraining

\begin{tabular}{|c|c|c|c|c|c|c|c|c|c|}
\hline \multirow[b]{3}{*}{ Test } & \multicolumn{9}{|c|}{ Effect size } \\
\hline & \multicolumn{3}{|c|}{ Within YO group } & \multicolumn{3}{|c|}{ Within 0 group } & \multicolumn{3}{|c|}{ Between groups } \\
\hline & 2 & 4 & 6 & 2 & 4 & 6 & 2 & 4 & 6 \\
\hline 8 foot up and go & 0.1 & 0.4 & 0.6 & 0.4 & 0.3 & 0.7 & 1.1 & 1.1 & 1.4 \\
\hline Chair stand & 0.3 & 0.4 & 0.4 & 0.6 & 0.7 & 0.7 & 0.6 & 0.8 & 1.1 \\
\hline Arm curl & 0.4 & 0.5 & 0.2 & 0.4 & 0.8 & 0.9 & 0.2 & 0.3 & 0.8 \\
\hline Six minute walk & 0.0 & 0.2 & 0.2 & 0.2 & 0.2 & 0.1 & 1.2 & 1.3 & 1.3 \\
\hline Chair sit and reach & 0.3 & 0.4 & 0.3 & 0.3 & 0.3 & 0.3 & 1.6 & 1.4 & 1.3 \\
\hline Back scratch & 0.1 & 0.2 & 0.1 & 0.1 & 0.2 & 0.1 & 1.0 & 1.0 & 1.0 \\
\hline
\end{tabular}

\section{RESULTS}

The YO group consisted of eight men and four women, and the $\mathrm{O}$ group consisted of nine men. Results from the two way, repeated measures analysis of variance indicated a significant main effect for time for the arm curl $\left(F_{4,76}=24.28\right.$, $\mathrm{p}<0.001)$, the chair stand $\left(F_{4,76}=68.79, \mathrm{p}<0.001\right)$, the up and go $\left(F_{4,76}=53.62, \mathrm{p}<0.001\right)$, the back scratch $\left(F_{4,76}=\right.$ $18.79, \mathrm{p}<0.001)$, the chair sit and reach $\left(F_{4,76}=16.58\right.$, $\mathrm{p}<0.001)$, and the six minute walk $\left(F_{4,76}=13.58, \mathrm{p}<0.001\right)$ tests. There were significant group effects for the up and go $\left(F_{1,19}=4.51, \mathrm{p}=0.047\right)$, chair sit and reach $\left(F_{1,19}=8.47\right.$, $\mathrm{p}=0.009)$, and six minute walk $\left(F_{1,19}=11.67, \mathrm{p}=0.003\right)$ tests (table 1).

After two weeks of detraining, none of the functional fitness test scores had changed in the YO group compared with immediately after training, whereas in the O group the chair stand and six minute walk test scores were significantly lower ( $\mathrm{p}=0.005$ and 0.006 respectively).

Scores on the arm curl $(p<0.001$ for YO, and $p=0.002$ for $\mathrm{O})$, chair stand $(\mathrm{p}<0.001$ for $\mathrm{YO}$, and $\mathrm{p}=0.001$ for $\mathrm{O})$, up and go $(\mathrm{p}=0.004$ for $\mathrm{YO}$, and $\mathrm{p}<0.001$ for $\mathrm{O})$, back scratch $(\mathrm{p}=0.001$ for $\mathrm{YO}$, and $\mathrm{p}<0.001$ for $\mathrm{O})$, chair sit and reach $(\mathrm{p}=0.002$ for $\mathrm{YO}$, and $\mathrm{p}=0.001$ for $\mathrm{O})$, and six minute walk $(\mathrm{p}<0.001$ for $\mathrm{YO}$, and $\mathrm{p}=0.002$ for $\mathrm{O})$ tests had declined at between two and four weeks of detraining.

In the YO group, there was significant loss in performance on the chair stand $(\mathrm{p}=0.004)$, chair sit and reach $(\mathrm{p}=$ $0.001)$, and six minute walk $(\mathrm{p}<0.001)$ tests at between four and six weeks of detraining. The chair stand and up and go test scores were significantly lower in the $\mathrm{O}$ group after six weeks of detraining than after four weeks of detraining $(\mathrm{p}<0.001$ and $\mathrm{p}=0.002$ respectively $)$.

In both groups, functional fitness test scores were significantly lower after six weeks of detraining than after the nine weeks of training $(\mathrm{p}<0.01)$, except the six minute walk test in the YO group $(\mathrm{p}=0.012)$. Performance in the chair stand test for both groups $(\mathrm{p}<0.001$ for $\mathrm{YO}$, and $\mathrm{p}=$ 0.006 for $\mathrm{O})$ and in the up and go test $(\mathrm{p}=0.003)$ and six minute walk test $(p=0.002)$ for the YO group remained significantly higher than before training after six weeks of detraining (table 1).

As shown in table 2, small to moderate effect sizes were observed after two weeks of detraining on all of the functional fitness tests in both of the groups. After four and six weeks of detraining, the effect sizes were small to moderate for both of the groups except the arm curl test for the $\mathrm{O}$ group. The largest between-effect sizes were observed for the chair sit and reach test after two and four weeks of detraining and for the up and go test after six weeks of detraining.

\section{DISCUSSION}

This study was designed to evaluate the effects of six weeks detraining on functional fitness in young-old and old people, and age responses to detraining in elderly people living independently.

The findings show that age affected performance loss on the up and go, six minute walk, and chair sit and reach tests during the six weeks of detraining. There were no significant losses on any of the functional fitness tests in the YO group, but performances in the chair stand and six minute walk tests for the O group had declined significantly from the posttraining values after two weeks of detraining. Scores on all of the functional fitness tests declined further between two and four weeks of detraining in both of the groups. In the YO group, the chair stand, chair sit and reach, and six minute walk test scores and in the O group, and the chair stand and up and go test scores were significantly lower after six weeks of detraining than after four weeks of detraining. However, performance in the chair stand test for both groups and in the six minute walk and up and go tests for the YO group remained significantly higher than before training after six weeks of detraining.

To our knowledge, this is the first study to compare the effects of age on the responses of functional fitness to detraining in elderly people. Lemmer et al ${ }^{8}$ compared young subjects (aged 20-30) with older subjects (aged 65-75) and found that age affected strength changes between 12 and 31 weeks of detraining, with greater decreases in strength in the older subjects than the young subjects. Häkkinen et $a l^{10}$ reported that only minor changes occurred in maximal strength, explosive jumping, and walking performances during three weeks of detraining in middle aged and older people. Ivey et al $^{11}$ investigated the effects of age on the loss of muscular strength during 31 weeks of detraining and stated that muscle quality remained significantly above baseline levels in young men and women (aged 20-30) and older men

\section{What is already known on this topic}

It is known that gains in cardiovascular fitness achieved during training last longer than gains in muscle strength in elderly people. However, no studies have compared the effects of detraining on functional fitness nor whether such effects differ with age in elderly people.

\section{What this study adds}

Loss of muscle strength and aerobic endurance begins earlier in the older elderly. Changes in flexibility, agility, and aerobic endurance performances in response to detraining are affected by age in elderly adults. Gains in aerobic endurance last longer in the younger elderly. 
(aged 65-75), but not older women. Although the protocols of these previous studies were different from that of our study, our finding that lower extremity strength performances remained significantly higher than before training is consistent with those studies. ${ }^{8} 1011$

In contrast, our finding that upper extremity strength reverted to pre-training values in both age groups after six weeks of detraining differs from data reported previously. ${ }^{13-15}$ It is difficult to explain the discrepancy between the upper and lower body response to six weeks of detraining in this small sample. However, detraining induced decreases in the arm curl performance may be related to the duration and type of the preceding training. It is possible that the duration of training and the multicomponent nature of the training programme, directed mostly at the lower extremities, used in this study caused more of a detraining induced decrease in the arm curl performance. The duration of training has been suggested to be an important contributing factor to the retention of neuromuscular adaptations once training has ended..$^{12}{ }^{18}$ Duration of training was longer ${ }^{14}{ }^{15}$ and intensity of training greater ${ }^{93-15}$ in the previous studies.

Decreased flexibility is significantly associated with the development of musculoskeletal impairments and the progression of disabilities in the elderly. ${ }^{19}$ However, the effect of detraining on the retention of flexibility in the elderly has not been adequately dealt with. Our finding of a large betweengroup effect size for the chair sit and reach test after two and four weeks of detraining suggests different rates of change in the two age groups. These data also imply that even short interruptions in regularly performed exercise may result in complete loss of training induced improvements in flexibility.

Various studies have indicated that performance on combined agility/dynamic balance tasks is a predictor of recurrent falling, ${ }^{2021}$ and physical exercise is an important factor in maintaining agility and balance. ${ }^{2}$ The large betweengroup effect size for the up and go test in our study suggested that agility and dynamic balance differed between the two groups after six weeks of detraining.

The YO group retained their recently acquired six minute walk performance after six weeks of detraining, although the six minute walk performance declined to pre-training values in the O group. The different responses between our YO and O subjects may reflect different adaptive processes. Sforzo et $a l^{7}$ reported that elderly people did not suffer any great impairment in cardiovascular exercise performance after 10 weeks of detraining. However, their training period was longer and the participants were fitter and had fewer medical conditions than our subjects. ${ }^{4}$

In summary, the results of this study show that age does influence the changes in agility/dynamic balance, lower extremity flexibility, and aerobic endurance during six weeks of detraining. However, six weeks of detraining does not reverse the gains in aerobic endurance and agility made during a nine week exercise programme in young-old (aged 60-73 years) adults and the gains in lower body strength of young-old and old (aged 74-86 years) people.

\section{ACKNOWLEDGEMENTS}

This study was supported by the Akdeniz University Research Foundation (No 20.01.0122.07(, the Akdeniz University Institute of Health Science, and Akdeniz University School of Physical Education and Sports. We thank Gülşah Sahin, Vedat Çetinkaya, and Burak Aglamıs for assistance with the conduct of this study. Special thanks go to all the subjects who volunteered.

\section{Authors' affiliations}

N F Toraman, N Ayceman, Akdeniz University School of Physical Education and Sports, Antalya, Turkey
Competing interests: none declared

\section{REFERENCES}

1 Rikli RE, Jones CJ. Senior fitness test manual. Champaign: Human Kinetics, 2001.

2 Rikli RE, Jones CJ. Functional fitness normative scores for community-residing older adults, ages 60-94. J Aging Phys Activ 1999;7:162-81.

3 Toraman NF, Erman A, Agyar E. Effects of multi-component training on functional fitness in older adults. J Aging Phys Act 2004;12:538-53.

4 Toraman F, Sahin G. Age responses to multicomponent training programme in older adults. Disabil Rehabil 2004;26:448-54.

5 Cavani V, Mier CM, Musto AA, et al. Effects of a 6-week resistance-training program on functional fitness of older adults. J Aging Phys Act 2002; 10:443-52.

6 Dudley GA, Snyder LLP. Deconditioning and bed rest: musculoskeletal response. In: Roitman JL, ed. ACSM's resource manual. Philadelphia: Lippincott Williams \& Wilkins, 1998:200-5.

7 Sforzo GA, McManis BG, Black D, et al. Resilience to exercise detraining in healthy older adults. J Am Geriatr Soc 1995;43:209-15.

8 Lemmer JT, Hurlbut DE, Martel GF, et al. Age and gender responses to strength training and detraining. Med Sci Sports Exerc 2000;32:1505-12.

9 Taeffe DR, Marcus R. Dynamic muscle strength alterations to detraining and retraining in elderly men. Clin Physiol 1997;17:311-24.

10 Häkkinen $K$, Alen $M$, Kallinen $M$, et al. Neuromuscular adaptation during prolonged strength training, detraining and re-strength-training in middleaged and elderly people. Eur J Appl Physiol 2000;83:51-62.

11 Ivey FM, Tracy BL, Lemmer JT, et al. Effects of strength training and detraining on muscle quality: age and gender comparisons. J Gerontol A Biol Sci Med Sci 2000;55:B152-9.

12 Smith K, Winegard K, Hicks AL, et al. Two years of resistance training in older men and women: the effects of three years of detraining on the retention of dynamic strength. Can J Appl Physiol 2003;28:462-74.

13 Elliott KJ, Sale C, Cable NT. Effects of resistance training and detraining on muscle strength and blood lipid profiles in postmenopausal women. $\mathrm{Br} J$ Sports Med 2002:36:340-4.

14 Lexell J, Downham DY, Larsson Y, et al. Heavy-resistance training in older Scandinavian men and women: short- and long-term effects on arm and leg muscles. Scand J Med Sci Sport 1995;5:329-41.

15 Porter MM, Nelson ME, Fiatarone Singh MA, et al. Effects of long-term resistance training and detraining on strength and physical activity in older women. J Aging Phys Act 2002;10:260-70.

16 Connely DM, Vandervoort AA. Effects of detraining on knee extensor strength and functional mobility in a group of elderly women. J Orthop Sports Phys 1997;26:340-6.

17 Rikli RE, Jones CJ. Development and validation of a functional fitness test for community-residing older adults. J Aging Phys Act 1999;7:129-61.

18 Hortobagyi T, Houmard JA, Stevenson JR, et al. The effects of detraining on power athletes. Med Sci Sports Exerc 1993;25:929-35.

19 Holland GJ, Tanaka K, Shigematsu R, et al. Flexibility and physical functions of older adults: a review. J Aging Phys Act 2002;10:169-206.

20 Podsiadlo D, Richardson S. The timed "up and go": a test of basic functional mobility for frail elderly persons. J Am Geriatr Soc 1991;39:142-8.

21 Tinetti ME, Williams TF, Mayewski R. Fall risk index for falls for elderly patients based on number of chronic conditions. Am J Med 1986;80:429-34.

\section{COMMENTARY}

This study evaluates the effect of six weeks of detraining on the fitness of elderly subjects, after a nine week exercise training programme. The subjects were classified as youngold (aged 60-73 years) or old (aged 74-86 years).

Functional fitness parameters such as agility/dynamic balance, lower extremity flexibility, and aerobic endurance seem to be influenced by age subgroups in the elderly. Upper extremity strength reverted to pre-training levels after six weeks in both age groups. Flexibility may be lost after a short interruption of the exercise programme. Aerobic endurance, lower body strength, and agility gains in the younger elderly and lower body strength gains in the older elderly did not reverse after a nine week training programme. These findings may encourage older adults to participate more regularly in exercise activities.

H Yaman

University of Akdeniz, Faculty of Medicine, Department of Family Medicine, Antalya, Türkiye; hakanyaman@akdeniz.edu.tr 